\title{
Assessment of research waste part 1: an exemplar from examining study design, surrogate and clinical endpoints in studies of calcium intake and vitamin D supplementation
}

\author{
Mark J. Bolland ${ }^{1 *}$, Alison Avenell ${ }^{2}$ and Andrew Grey ${ }^{1}$
}

\begin{abstract}
Background: Research waste is estimated to be very common, but assessments of its prevalence and scope are rare. As an example, we assessed research waste in clinical research on calcium intake (assessing study design and endpoint type) and vitamin D supplementation (assessing endpoint type).
\end{abstract}

Methods: We examined 404 randomised controlled trials (RCTs) and observational studies of calcium intake (diet or supplements) and bone mineral density (BMD) or fracture, and 547 RCTs of vitamin D supplements, and assessed the proportion of studies that used surrogate or clinical endpoints. For studies with BMD or fracture as an endpoint, we estimated when the 'tipping' point occurred indicating the need for RCTs with fracture as an endpoint (based on cumulative meta-analyses of BMD RCTs, and chronological review of observational studies), and whether each study published at least $5 y$ after the tipping point was novel, added new clinical knowledge or was research waste.

Results: Observational studies of calcium intake and BMD or fracture outnumbered RCTs by 3.3-4.5 times. For both calcium intake and vitamin D supplements, studies using surrogate endpoints outnumbered studies using clinical endpoints by 1.6-3 times. Of 41 RCT publications of calcium intake and BMD or fracture published at least $5 y$ after the tipping point in 1994, we considered that 19 (46\%) lacked novelty, another 13 (32\%) added no new clinical knowledge, and 30 (73\%) were research waste. Of 204 observational study publications of calcium intake and BMD or fracture, 197 (96\%) lacked novelty, another 5 (2\%) added no new clinical knowledge, and 202 (99\%) were research waste. Of $39 \mathrm{RCT}$ s of vitamin $\mathrm{D}$ supplementation and BMD or fracture published at least $5 \mathrm{y}$ after the tipping point in 1999, 14 (36\%) lacked novelty, another 13 (33\%) added no new clinical knowledge, and 27 (69\%) were research waste.

Conclusions: A high proportion of studies of calcium intake since 2000 (95\%) and trials of vitamin D supplements since 2005 (69\%) on BMD or fracture represent research waste.

Keywords: Calcium intake, Vitamin D, Research waste, Observational studies, Randomized controlled trials, Surrogate endpoints, Bone density, Fracture

\footnotetext{
* Correspondence: m.bolland@auckland.ac.nz

${ }^{1}$ Bone and Joint Research Group, Department of Medicine, University of

Auckland, Private Bag 92 019, Auckland 1142, New Zealand

Full list of author information is available at the end of the article
}

(c) The Author(s). 2018 Open Access This article is distributed under the terms of the Creative Commons Attribution 4.0 International License (http://creativecommons.org/licenses/by/4.0/), which permits unrestricted use, distribution, and reproduction in any medium, provided you give appropriate credit to the original author(s) and the source, provide a link to the Creative Commons license, and indicate if changes were made. The Creative Commons Public Domain Dedication waiver (http://creativecommons.org/publicdomain/zero/1.0/) applies to the data made available in this article, unless otherwise stated. 


\section{Background}

Redundant research represents a large cost to society and misuses participants' resources and altruism. Chalmers and Glasziou estimated that more than $85 \%$ of research might be wasteful because the wrong research questions are studied or because of poorly designed studies, inaccessible results, and biased reporting [1]. Very few studies have systematically examined research topics for evidence of waste, or established the methodology for doing so. In these companion reports [2], we assessed research waste in a single field - calcium and vitamin D research.

Calcium intake (through diet or supplements) and vitamin D supplementation have been very extensively researched as interventions for older adults for over 30 years. While undertaking recent systematic reviews [3-8], we noticed that many studies in these fields seemed to be designed to replicate existing knowledge rather than to determine new clinically relevant findings. The large body of published research in these fields allows an assessment of one important aspect of research waste, the quantity of research conducted that was unnecessary because existing knowledge was extensive. In this first report, we use existing databases compiled from our recent meta-analyses, which collated all studies of increased calcium intake and all randomised controlled trials (RCTs) of vitamin D supplements. We assess whether each study with an endpoint of bone mineral density (BMD) or fracture constitutes research waste, i.e. an unnecessary duplication without addressing shortcomings in previous research [9]. We focus on study design (observational versus RCT) in calcium intake studies and type of endpoint (surrogate versus clinical) in vitamin D supplementation RCTs.

\section{Methods}

\section{Literature search and study selection}

Calcium intake studies

The literature search has previously been published and is described in full in the Additional file 1: Supplementary Methods, Table S1, Figure S1 [7, 8]. Briefly, we identified 404 RCTs and observational studies (cohort, case-control, or cross-sectional studies) published before September 2014 of calcium, milk, or dairy intake, or calcium supplements with fracture or BMD as outcomes in participants $>50$ years who had no major systemic illnesses other than osteoporosis. As a secondary analysis, we also assessed studies with cardiovascular and cancer endpoints.

\section{Vitamin D supplementation RCTs}

The search is described in full in the Additional file 1: Supplementary Methods, Tables S2-S3, Figure S2. Briefly, we identified 547 RCTs of vitamin D supplements in adults (>18y) published before December 2015 and classified them by the clinical relevance of their endpoints.
We categorised each RCT according to whether clinical or surrogate endpoints were reported in the Abstract, using the Institute of Medicine's definition of surrogate outcomes as "biomarker[s] intended to substitute for a clinical endpoint [and] expected to predict clinical benefit (or harm ...) based on epidemiologic, therapeutic, pathophysiologic, or other scientific evidence" [10]. If there were multiple publications or endpoints, we included the study report with the most relevant clinical endpoint, or if there were no clinical endpoints, the study report with the most clinically relevant surrogate endpoint (Table 1).

Table 1 Classification of endpoints in 547 randomised controlled trials of vitamin D supplementation

\begin{tabular}{|c|c|}
\hline Endpoint $^{\mathrm{a}}$ & Number of RCTs \\
\hline \multicolumn{2}{|l|}{ Clinical endpoints } \\
\hline Fracture & 18 \\
\hline Falls & 17 \\
\hline Respiratory (eg asthma, COPD, URTI) & 14 \\
\hline Musculoskeletal symptoms/Pain & 11 \\
\hline Pregnancy outcomes & 9 \\
\hline Tuberculosis & 8 \\
\hline Multiple Sclerosis & 7 \\
\hline Mood & 6 \\
\hline SLE/Rheumatoid Arthritis & 6 \\
\hline Other & 41 \\
\hline \multicolumn{2}{|l|}{ Surrogate endpoints } \\
\hline Bone mineral density & 57 \\
\hline $25 \mathrm{OHD}$ only & 49 \\
\hline HbA1c or measures of glycaemia & 42 \\
\hline Blood pressure & 38 \\
\hline Basic biochemistry & 37 \\
\hline Bone turnover markers & 31 \\
\hline $25 \mathrm{OHD}$, vitamin D metabolites and/or PTH only & 24 \\
\hline Muscle Strength & 16 \\
\hline Body weight & 15 \\
\hline Physical performance tests & 14 \\
\hline Vascular properties & 7 \\
\hline Hepatitis C viral load & 5 \\
\hline Lung function tests & 4 \\
\hline Lipids & 4 \\
\hline Other laboratory tests/assays & 51 \\
\hline Other endpoints & 16 \\
\hline
\end{tabular}

Abbreviations: COPD chronic obstructive pulmonary disease, URTI upper respiratory tract infection, SLE Systemic lupus erythematosus, 25OHD 25 hydroxyvitamin $\mathrm{D}, \mathrm{PTH}$ parathyroid hormone

${ }^{a}$ many trials reported more than 1 endpoint in the abstract. The most clinically relevant endpoint was used for each study 


\section{Determination of research waste}

For observational studies of calcium intake, we examined the results of publications chronologically and by consensus (MB, AG) determined the 'tipping' point - the time at which the hypothesis that calcium intake was associated with BMD or fracture had been generated and warranted testing in RCTs. For RCTs of calcium intake or vitamin D supplements, we conducted cumulative meta-analyses for BMD, a surrogate endpoint for the clinical endpoint of fracture. These meta-analyses were based on previous systematic reviews of calcium intake (with or without vitamin D supplements) and BMD [8] and vitamin D supplements (used as monotherapy, such that treatment groups only differed by vitamin $\mathrm{D}$ use) on BMD [6]. We then determined by consensus (MB, AG) if a 'tipping' point occurred: that is, the time at which the treatment effect was established and RCTs were required to investigate efficacy for the clinically meaningful outcome of fracture. For each category of research, we allowed a 5 year period after the tipping point, for publication of research in progress and dissemination and uptake of existing knowledge.

Each subsequent publication was independently reviewed by two authors (MB, AG). Firstly, we determined whether its study design was novel, and secondly whether it added to existing clinical knowledge and then, taking into account both these factors in a stepwise fashion, whether it represented research waste. These assessments were not based on study results. We considered a study novel when the population group, intervention, or dose did not overlap with existing publications, or there were potentially important novel features in the study design. Studies not considered novel were classified as research waste. We considered a study added to existing clinical knowledge if, by its design, it could produce results that might potentially modify the existing conclusion that RCTs with fracture as an endpoint were required. Studies that did not add to existing knowledge were considered research waste. Thus, a study could be classified as novel (e.g. a previously unstudied population) but if it could not add to existing knowledge, it was classified as research waste. Assessments were performed independently and any disagreements resolved by discussion. Agreement between assessors for novelty was 69-92\%, for addition of knowledge 80$98 \%$, and for waste $76-98 \%$.

\section{Statistics}

Cumulative meta-analyses were performed using random effects meta-analyses based on previously published data $[6,8]$ using Comprehensive Meta-Analysis (Version 2, Biostat, Englewood New Jersey, USA). P-values $<0.05$ were considered statistically significant.

\section{Results \\ Calcium intake studies Study design}

Figure 1 shows the number of publications of calcium intake with BMD or fracture as an endpoint over time, by endpoint and study design. Observational studies outnumber RCTs by 3.3-4.5 times, and studies with BMD as an endpoint outnumber studies with fracture as an endpoint by 1.6-2.2 times. By 2014, the number of RCTs with either endpoint had plateaued, while the number of observational studies continued to steadily increase. For example, between 2009 and 13, there were 43 publications from observational studies and 8 from RCTs with BMD as an endpoint, and 31 publications from observational studies and 3 from RCTs with fracture as an endpoint.

Table 2 shows the secondary analysis results with the number of publications of calcium intake with clinical non-skeletal endpoints by study design. Observational studies outnumber RCTs by 3.4-43 times. Despite the very large number of observational studies, only two RCTs had a primary non-skeletal endpoint.

\section{Determination of tipping point}

We performed cumulative meta-analyses of the effect of increasing calcium intake on lumbar spine (Fig. 2) or femoral neck BMD (Fig. 3) in RCTs at $1 y$ and $2 y$. By the end of 1994, the meta-analyses show statistically significant increases in BMD at the lumbar spine of 1.4\% (95\% CI $0.3,2.6)$ at $1 y$ from 7 RCTs involving 792 participants, and $1.4 \%(95 \% \mathrm{CI} 0.7,2.2)$ at $2 \mathrm{y}$ from 8 RCTs involving 852 participants. Likewise, by this time the pooled effect was $1.4-1.8 \%$ at the femoral neck at $1-2 y$ from 5 RCTs involving 450-471 participants. The pooled effect sizes change little with the addition of data from later RCTs. By the end of 1994, RCTs had been conducted in most clinically relevant populations, including early and late post-menopausal women, men, people with recent fractures, different ethnic populations, and cohorts with low calcium intake. In addition, different doses of calcium supplements, different supplements, and milk products had all been evaluated in RCTs (Additional file 1: Table S4).

The first cohort study with more than 100 fractures and 10,000 participants was published in 1991, and by the end of 1994 there were 6 publications from cohort studies involving $>28,000$ participants with $>600$ hip fractures. By this time, 24 observational studies (6 cohort, 18 case-control, and 2 cross-sectional studies) had reported on the relationship between dietary calcium, dairy or milk intake or calcium supplements and fracture in cohorts with a wide variety of clinical characteristics.

We therefore defined 1994 as the tipping point for calcium intake and fractures. By this time, observational studies had reported that calcium intake might be 


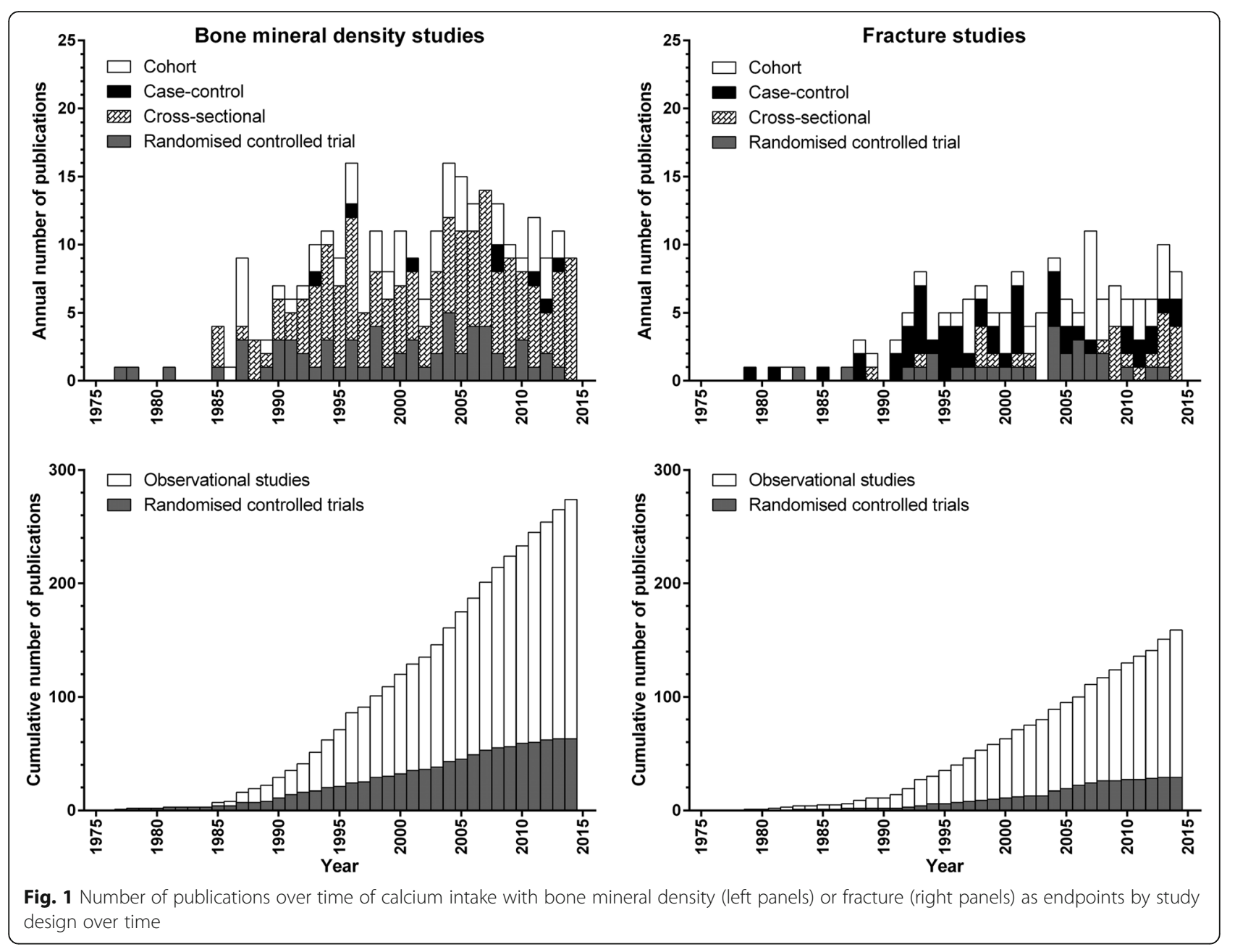

Table 2 Publications of calcium intake with non-skeletal endpoints by study design

\begin{tabular}{lllllll}
\hline Endpoint & \multicolumn{3}{l}{ Observational studies } & \multicolumn{3}{l}{ RCT $^{a}$} \\
\cline { 2 - 5 } & Cross-sectional & Case-control & Cohort & Total & \\
\hline IHD/MI & 1 & 4 & 27 & 31 & $9^{\mathrm{b}}$ \\
Stroke & 1 & 2 & 25 & 27 & $8^{\mathrm{b}}$ \\
Cancer (any site) & 0 & 188 & 112 & 300 & $7^{\mathrm{c}}$ \\
Colorectal cancer & 0 & 49 & 45 & 94 & $3^{\mathrm{c}}$ \\
Breast cancer & 0 & 25 & 25 & 50 & $3^{\mathrm{c}}$ \\
Prostate cancer & 0 & 34 & 32 & 66 & $2^{c}$ \\
Colorectal adenoma & 3 & 14 & 8 & 25 & 2
\end{tabular}

Abbreviation: IHD/MI ischaemic heart disease or myocardial infarction, $R C T$ randomised controlled trial

${ }^{a}$ Two RCTs had colorectal adenoma as the primary endpoint. All other RCTs reported data as secondary endpoints

bUnpublished data for myocardial infarction and stroke from an additional 11 RCTs published in meta-analyses $[25,26]$

'Unpublished data for cancer published in a meta-analysis [27] from an additional 6 RCTs for total cancer and colorectal cancer, 4 RCTs for breast cancer, and 2 RCTs for prostate cancer associated with fracture risk, and RCTs had confirmed that increasing calcium intake increased the surrogate endpoint of BMD. Thus, further RCTs with BMD as an endpoint or observational studies with either BMD or fracture endpoints were unnecessary unless there was a novel hypothesis to be tested. Allowing $5 y$ for dissemination of this information, we considered whether publications from 2000 onwards represented research waste.

\section{Estimating research waste}

Of 41 RCTs publications from 2000 onwards (Additional file 1: Table S4), 19 reported fracture data. Seven RCTs had a primary endpoint of fracture and were considered novel and not waste. One publication was a novel exploratory analysis of a previous RCT which had fracture as the primary endpoint, but did not add new clinical knowledge and we considered it research waste. Three RCTs with other novel primary endpoints reported fracture data as a secondary endpoint, but none added new clinical knowledge because of their small size and few fracture events. We considered 1 of the 3 publications to be research waste because it did not report the primary 




endpoint of the study, instead being a secondary publication that only reported fracture data. The major bone-related focus of the remaining 30 RCTs was BMD, either as the primary $(n=23)$ or secondary $(n=7)$ endpoint. Eight of these $30 \mathrm{RCTs}$ reported fracture data as a secondary endpoint. Of these $30 \mathrm{RCTs}$, we considered 11 novel (8 novel study design, 2 novel population groups, 1 novel dose/population), but only 2 of these 11
RCTs added new clinical knowledge, and we considered that 28 of the 30 RCTs were research waste. Thus, of the $41 \mathrm{RCT}$ publications at least $5 \mathrm{y}$ after the tipping point, 19 (46\%) lacked novelty, another 13 (32\%) did not add new clinical knowledge, and overall we considered 30 (73\%) of these RCT publications were research waste. Twenty-four of the 30 reports were primary publications of RCTs ( $n=4537$ participants).

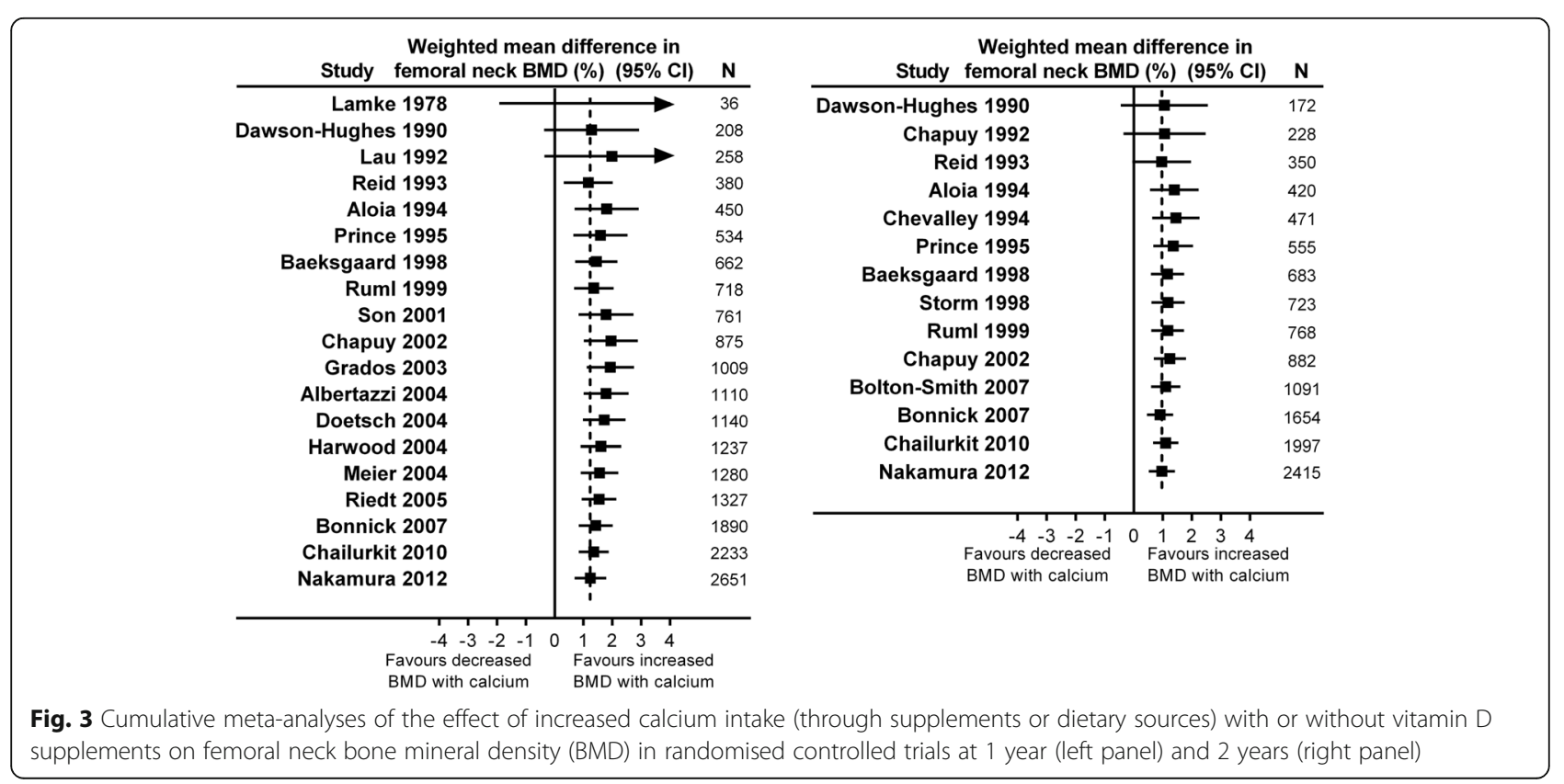


Of 204 observational study publications from 2000 onwards, 133 reported data on calcium intake and BMD, and 82 on fracture (Additional file 1: Table S5). In 51, 26 , and 10 publications respectively, a primary purpose was to examine the relationship between calcium intake and BMD, or fracture, or both BMD and fracture. In the other 82, 56, and 1 publications respectively, examining calcium intake was a secondary hypothesis, with the primary purpose to either investigate the determinants of $\mathrm{BMD}$, or fracture risk, or both BMD and fracture risk, or to examine the relationship between a specific factor and these endpoints. We considered that $4 / 133$ publications reporting BMD relationships and 3/82 reporting fracture relationships were novel. In 1 publication considered a novel study design, the relationship between calcium intake and BMD was the primary focus. In the other 6 publications, the relationship between calcium intake and BMD or fracture was only a secondary focus: 2 examined determinants of fracture and 2 determinants of BMD in previously unreported clinical situations, and 2 developed predictive models for BMD or fracture. We considered that 2 of these 7 papers added new clinical knowledge, whereas the other 5 were research waste. Thus, of the 204 observational study publications from 2000 onwards, 197 (96\%) lacked novelty, another 5 (2\%) did not add new clinical knowledge, and overall we considered 202 (99\%) were research waste. Of the 67 publications with a primary focus on calcium intake and BMD or fracture, only 1 was novel, and all were considered research waste.

\section{Vitamin D supplementation randomised controlled trials Study classification by endpoint}

Figure 4 shows the number of RCTs with clinical endpoints in the abstract $(n=137)$ compared with RCTs with surrogate but no clinical endpoints in the abstract $(n=410)$. The RCTs with surrogate endpoints are further subdivided into those that only report concentrations of 25-hydroxyvitamin $D$, other vitamin D metabolites, and/or parathyroid hormone in the abstract $(n=73)$ and those that report other endpoints $(n=337)$. There has been a steady increase in the number of RCTs published over time, particularly since

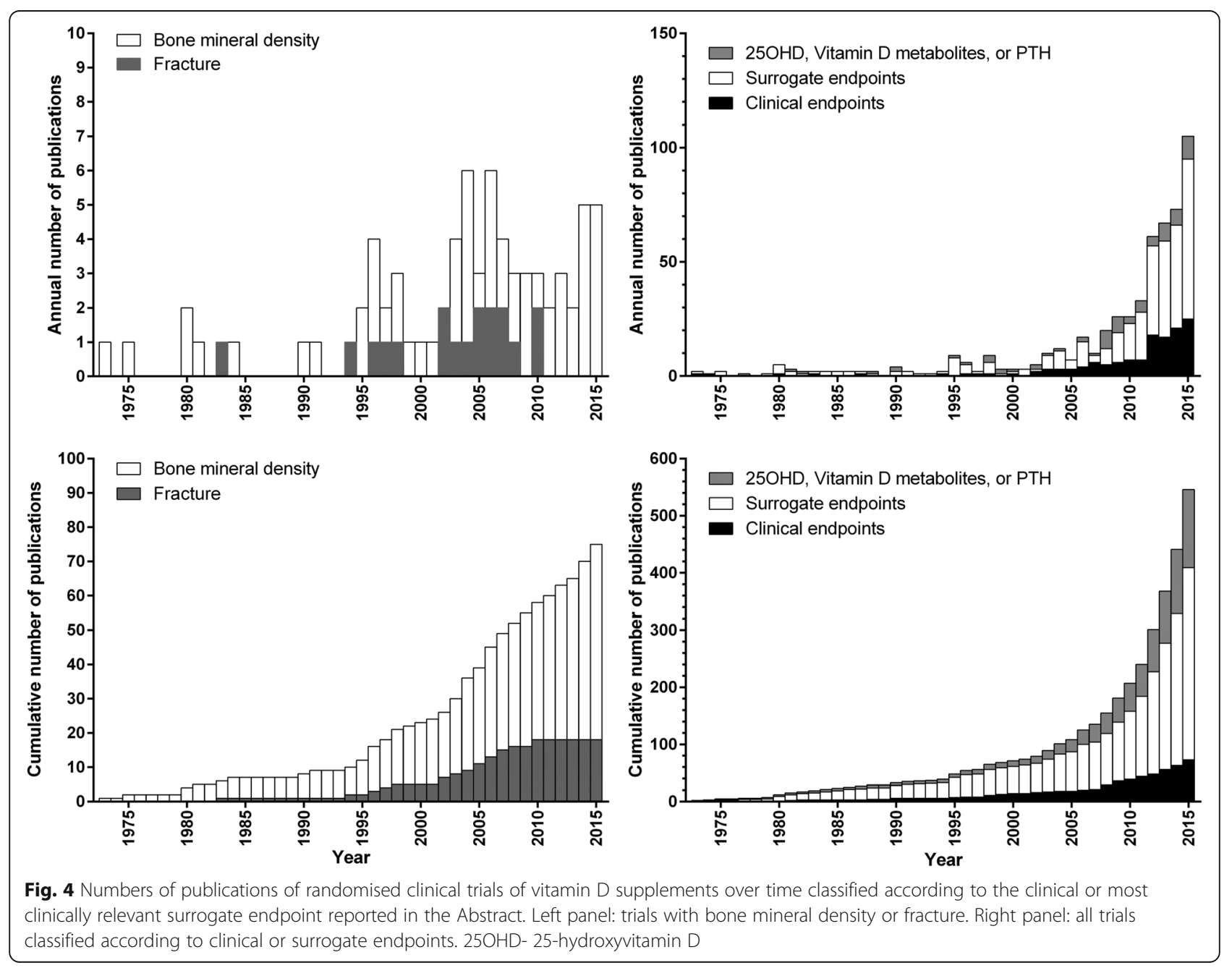


2005. Since 2012, there have been more than 50 RCTs published each year. RCTs with surrogate endpoints outnumber RCTs with clinical endpoints by 3 times. In RCTs with skeletal endpoints, RCTs of the surrogate endpoint, BMD $(n=57)$, outnumber RCTs with the clinical endpoint of fracture $(n=18)$ by 3.2 times.

\section{Determination of tipping point}

Figure 5 shows cumulative meta-analyses of the effect of vitamin $\mathrm{D}$ supplements at $1-2 \mathrm{y}$ on lumbar spine and femoral neck BMD in RCTs. By the end of 1999, the meta-analyses show stable effect sizes for BMD at each site from 4 RCTs involving $>900$ participants. By this time, vitamin $\mathrm{D}$ supplements had no effect on lumbar spine BMD $(n=918$, RR $0.3 \%, 95 \%$ CI $-0.1,0.8)$, but increased femoral neck BMD by 1.0\% (95\% CI 0.1, 2.0, $n=$ 975). The pooled effect sizes change little with the addition of data from later RCTs. We therefore defined 1999 as the tipping point and, allowing 5y for dissemination of this information, we considered whether publications from 2005 onwards represented research waste.

\section{Estimating research waste}

Thirty-nine RCTs of vitamin D supplements with the most clinically relevant endpoint of BMD or fracture were published from 2005 onwards (Additional file 1: Table S6). In 9, the primary endpoint was fracture and all were considered novel and not waste. Of the remaining $30 \mathrm{RCTs}$ that reported BMD data, we considered that 16 were novel because they included novel designs $(n=6)$, or populations $(n=10)$, but only 3 of these 16 studies added new clinical knowledge, and we considered that 27 of the 30 RCTs were research waste. Thus, of the 39 RCTs published at least $5 y$ after the tipping point was reached, 14 (36\%) were not novel, another 13 (33\%) did not add new clinical knowledge, and overall we considered $27(69 \%)$ of these RCTs $(n=3264$ participants) were research waste.

\section{Discussion}

These results, obtained in a specific field of research, support the view of Chalmers and Glasziou that a very high proportion of published research is wasteful. For both calcium intake and vitamin D supplementation, both observational studies and RCTs with surrogate endpoints were far more prevalent than RCTs with clinical endpoints. Thus, observational studies of calcium intake outnumbered RCTs by at least 3 times, and for both calcium intake and vitamin D supplementation, studies using surrogate endpoints outnumbered studies using clinical endpoints by 1.6-3 times. For the skeletal endpoints of BMD and fracture, these imbalances persisted long after the tipping point when the hypothesis that calcium intake or vitamin D supplementation might alter fracture risk had been addressed and answered. Of RCTs of calcium intake or vitamin D supplementation and fracture or BMD published at least $5 y$ after the tipping point, $36-46 \%$ were not novel, testing hypotheses about effects on BMD that had previously been established. Of those considered novel, $60-80 \%$ added no new clinical knowledge, and amounted to a third of the published RCTs. Almost all observational studies of calcium intake and BMD or fracture published $5 \mathrm{y}$ after the tipping point were not novel. Thus, we considered that since 2000, $73 \%$ of RCTs, $98 \%$ of observational studies, and $95 \%$ of clinical research on calcium intake and BMD or fracture was wasteful and that since $2005,69 \%$ of RCTs of vitamin D supplements and BMD or fracture

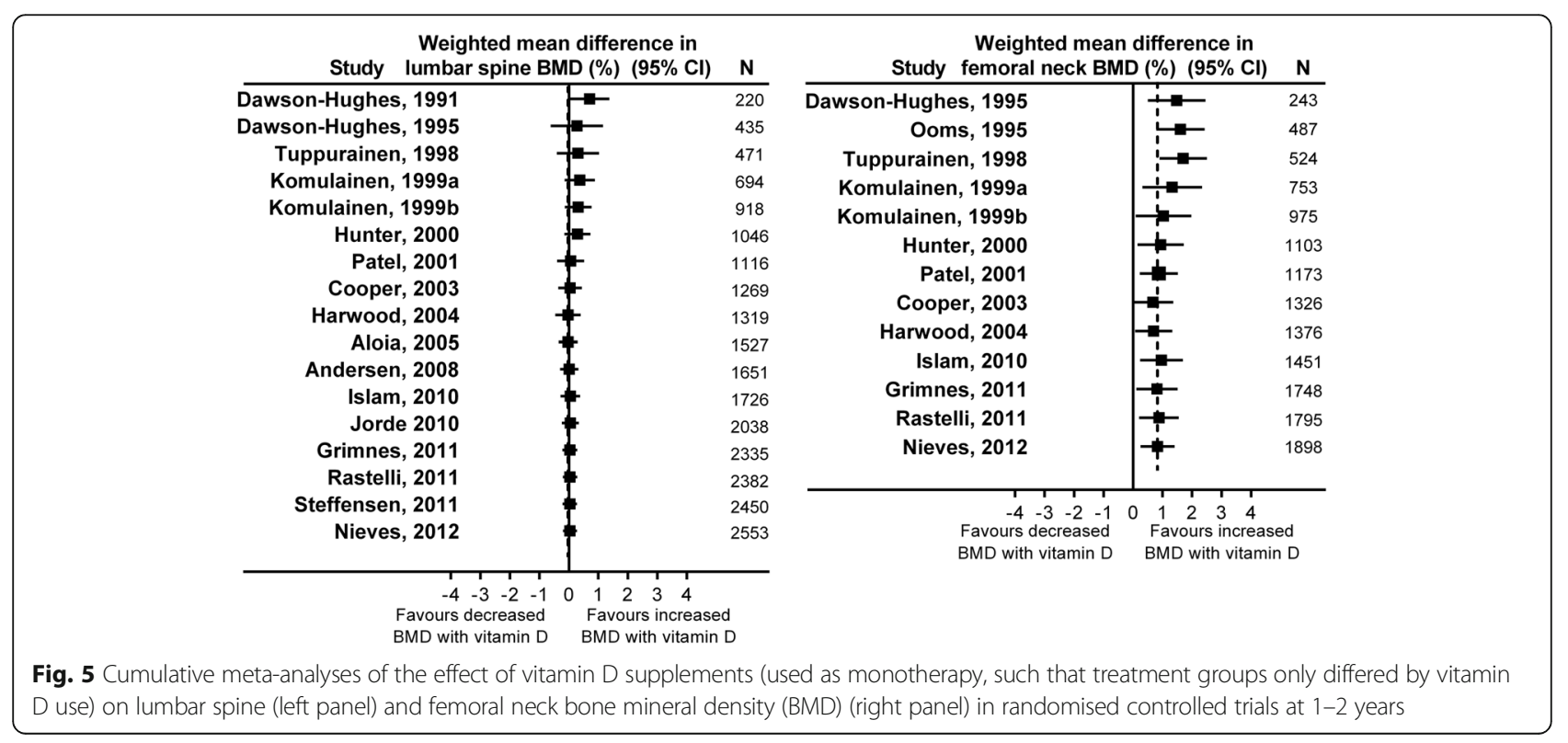


were research waste. In research on non-skeletal endpoints, the marked imbalances between observational studies and RCTs, and between RCTs with surrogate and clinical endpoints suggest that waste in researching calcium and vitamin $\mathrm{D}$ is not restricted to bone health.

In estimating that $85 \%$ of research represents waste [1], Chalmers and Glasziou focused on four aspects - the choice of research questions, quality of research, unpublished results, and the quality of research reports. Our findings are relevant to the first and second aspects. Chalmers and Glasziou estimated that $50 \%$ of studies are wasteful because they are unnecessary, poorly designed without reference to a systematic review, and/or have major methodological weaknesses [1]. Our findings suggest that this may be a considerable underestimate, at least in some research areas. Studies with weaker designs (observational studies or RCTs with surrogate endpoints) were far more prevalent than RCTs with clinical endpoints, but we considered almost all (90-98\%) such studies published over a 15-20 year period to represent research waste. If our findings apply to other fields, the overwhelming majority of RCTs and observational studies published in mature research areas may be wasteful.

A strength of our study is the extensive systematic literature searches and categorisation of large, complete sets of observational studies and RCTs of calcium intake and skeletal health, and RCTs of vitamin D supplements published over a long period of time. We are not aware of previous attempts to categorise or quantify research waste, but our methods are very labour and time intensive, and require adequate resourcing if they were to be more widely utilised. Limitations include that only two research areas were studied and therefore the results might not apply to other fields, and that we estimated the scope but not cost of the waste. We also considered both study design and study endpoints, which we think makes any conclusions more compelling than if the research areas or the study design and endpoints were assessed separately. Assessing studies as novel, contributing to clinical knowledge, or representing research waste requires subjective decisions. In general, however, agreement between the independent assessments was high. Agreement was lowest for novelty because many studies were of highly selected cohorts not previously studied. Deciding whether small differences from previous cohorts (such as ethnicity, gender or age) are sufficiently unique to be considered novel is difficult. Replication of previous studies is essential, and while disagreements may occur as to whether a study represents replication or redundancy, the number of observational studies and RCTs with surrogate endpoints extended well beyond the number needed for replication. Research can take a long time from inception to completion, and it is possible that some studies were initiated before the tipping point but not published until more than $5 \mathrm{y}$ after the tipping point, and were then assessed as not contributing to current knowledge. The literature search includes papers published until September 2014. As a methodological study, it was an extremely comprehensive, but very time-consuming attempt to identify all relevant clinical studies (observational and RCT) for a large number of outcomes. This search took almost 12 months to complete, and so it was not practical to update it subsequently. We are not aware of an RCT of calcium or vitamin D with fracture as the primary endpoint reported since 2014. Based on the data from 2011 to 2014, we would expect more than 75 observational studies to have been published since 2014, but $<10$ RCTs of calcium and $>200$ RCTs of vitamin D supplements. These recent publications are therefore unlikely to alter our conclusions related to research waste in this field.

An important issue raised by this research is how to assess research waste. Widely accepted definitions of waste are not yet available, and an element of subjectivity will always be required. To minimise the impact of subjectivity, we followed approaches used for high quality evidence-based systematic reviews: we explicitly stated the criteria for studies to be categorised as research waste, independently assessed studies, documented levels of agreement, resolved disagreements by consensus discussion, and reported our judgements for each study. However, other researchers might classify studies differently, and differences of opinion are common in similar circumstances. For example, Cochrane researchers recently reported that $45 / 156$ risk of bias judgements differed between two groups of authors [11]. Differences in definitions and study classification between researchers might provide insight into this issue and lead to better definitions of research waste, and better approaches to its assessment.

A related issue is when the tipping point can be considered to have occurred. In the design phase of any $\mathrm{RCT}$, a literature review should be standard practice. Thus, trialists should be aware of the most recent evidence before the study protocol is finalised, and therefore whether RCTs with meaningful clinical endpoints should be undertaken, or whether more observational studies or RCTs with surrogate endpoints are still required. Researchers may have different views on the exact date the tipping point occurred, but the cumulative meta-analyses and publication rates of observational studies highlight that it was many years ago that the hypothesis of fracture prevention for both calcium and vitamin D supplementation was formulated. The exact date of the tipping point, and whether 5 years is sufficient to allow dissemination of that information may be open to debate, but, in our example, would not change 
the conclusion that the majority of recent published research has not addressed the issue of fundamental clinical importance- whether calcium or vitamin D supplementation prevents fractures.

Research waste and how it can be reduced is increasingly being considered an important topic [9, 12-19]. The suggestions most relevant to our findings are the improvement of research priorities [9] and study design [15], in particular, performing systematic reviews to inform research proposals, and ensuring that study methods are optimum. Our finding that research waste continues unabated for long periods of time after a hypothesis has been generated or a research question has been answered suggests that most academics and funders have not so far corrected this problem. Publication of all RCTs is recommended, so if research has progressed to the stage of publication, it is difficult to argue that the primary RCT results should not be published. Considerable onus therefore necessarily falls upon assessments performed at the funding or ethical approval stage. These should be informed by a systematic review $[9,20]$, but that may not be straightforward. If systematic reviews exist, they often have discordant findings [21], and "living" systematic reviews updated with new evidence in real-time are not yet available [22]. Conducting a systematic review is time-consuming but necessary if one is not available or out-of-date. Finding relevant research is difficult because bibliographic database searches are non-specific, require screening of very many titles and abstracts, and frequently miss relevant studies. Involvement of information scientists and methodological expertise in the appraisal of funding applications might help to reduce research waste. This would require investment in appropriate resources but might improve the efficiency and accuracy of research funding.

We found that a very high proportion of observational research on calcium intake was wasteful. Many observational analyses are conducted opportunistically [23], and journal reviewers and editors could play important roles in declining manuscripts that examine established hypotheses that need, or have already been tested in, randomised studies, or where the findings are fragile and unlikely to be correct [24]. However, the large number of journals courting publications and academic incentives for publication quantity suggest that this strategy may be difficult to realise.

\section{Conclusion}

We identified a very high proportion of research waste in the fields of calcium intake and vitamin D supplementation for bone health after the need for RCTs with meaningful clinical endpoints had been established. This waste was largely due to continued publication of observational studies and RCTs with surrogate endpoints unable to address the relevant clinical questions. Nearly all the observational studies and nearly half the RCTs were not novel and another third of RCTs added no new clinical knowledge. Thus, collectively, 95\% of recent RCT or observational studies of calcium intake and BMD or fracture, and $69 \%$ of recent RCTs of vitamin D supplements and BMD or fracture were research waste. Strategies to reduce research waste should be devised and studied to determine their effectiveness.

\section{Additional file}

Additional file 1: Supplementary methods. Table S1. Literature
searches for calcium intake studies. Table S2. Searches of Pubmed for vitamin
D randomised controlled trials undertaken in December 2015. Table S3.
Thirty-eight systematic reviews of vitamin D supplements identified in
Pubmed search. Table S4. Classification and characteristics of randomised
controlled trials of increased calcium intake and bone mineral density or
fracture. Table S5. Classification and characteristics of observational studies
of calcium intake and bone mineral density or fracture. Table S6. Classification
and characteristics of randomised controlled trials of vitamin D supplements
and bone mineral density or fracture. Figure S1. Flow of studies for calcium
intake. Figure S2. Flow of studies for randomised controlled trials of vitamin
D supplements. (DOCX $922 \mathrm{~kb}$ )

\section{Abbreviations}

BMD: bone mineral density; $R C T$ : randomised controlled trial

\section{Funding}

No specific funding was received for this study. MB receives salary support from the Health Research Council of New Zealand. The Health Services Research Unit is funded by the Chief Scientist Office of the Scottish Government Health and Social Care Directorates. The funders had no role in the study design; collection, analysis, and interpretation of the data; writing of the report; and in the decision to submit the paper for publication.

\section{Availability of data and materials}

All data generated or analysed during this study are included in this published article and its supplementary information file.

\section{Authors' contributions}

$M B, A G$ and $A A$ designed the research. $M B$ identified the studies from the previous literature search. MB and AG reviewed the studies. MB performed the analyses. MB drafted the paper. All authors critically reviewed and improved it. All authors read and approved the final manuscript.

Ethics approval and consent to participate

Not applicable.

\section{Consent for publication}

Not applicable.

\section{Competing interests}

$M B, A G$, and $A A$ have all published randomised controlled trials and systematic reviews in the fields of calcium and vitamin D but otherwise have no competing interests to declare.

\section{Publisher's Note}

Springer Nature remains neutral with regard to jurisdictional claims in published maps and institutional affiliations.

\section{Author details}

'Bone and Joint Research Group, Department of Medicine, University of Auckland, Private Bag 92 019, Auckland 1142, New Zealand. ${ }^{2}$ Health Services Research Unit, University of Aberdeen, Foresterhill, Aberdeen AB25 2ZD, Scotland. 
Received: 10 July 2018 Accepted: 10 September 2018

Published online: 10 October 2018

\section{References}

1. Chalmers I, Glasziou P. Avoidable waste in the production and reporting of research evidence. Lancet. 2009;374:86-9.

2. Bolland MJ, Grey A, Avenell A. Assessment of research waste part 2: wrong study populations- an exemplar of baseline vitamin D status of participants in trials of vitamin D supplementation. BMC Medical Research Methodology. https://doi.org/10.1186/s12874-018-0555-1.

3. Avenell A, Mak JC, O'Connell D. Vitamin D and vitamin D analogues for preventing fractures in post-menopausal women and older men. Cochrane Database Syst Rev. 2014;4:CD000227.

4. Bolland MJ, Grey A, Gamble GD, Reid IR. The effect of vitamin D supplementation on skeletal, vascular, or cancer outcomes: a trial sequential meta-analysis. Lancet Diabetes Endocrinol. 2014;2:307-20.

5. Bolland MJ, Grey A, Gamble GD, Reid IR. Vitamin D supplementation and falls: a trial sequential meta-analysis. Lancet Diabetes Endocrinol. 2014;2: 573-80.

6. Reid IR, Bolland MJ, Grey A. Effects of vitamin D supplements on bone mineral density: a systematic review and meta-analysis. Lancet. 2014;383: 146-55

7. Bolland MJ, Leung W, Tai V, Bastin S, Gamble GD, Grey A, et al. Calcium intake and risk of fracture: systematic review. BMJ. 2015;351:h4580.

8. Tai V, Leung W, Grey A, Reid IR, Bolland MJ. Calcium intake and bone mineral density: systematic review and meta-analysis. BMJ. 2015;351: h4183.

9. Chalmers I, Bracken MB, Djulbegovic B, Garattini S, Grant J, Gulmezoglu AM, et al. How to increase value and reduce waste when research priorities are set. Lancet. 2014;383:156-65.

10. IOM (Institute of Medicine). Evaluation of biomarkers and surrogate endpoints in chronic disease. Washington (DC): National Academies Press (US); 2010.

11. Jordan VM, Lensen SF, Farquhar CM. There were large discrepancies in risk of bias tool judgments when a randomized controlled trial appeared in more than one systematic review. J Clin Epidemiol. 2017;81:72-6.

12. Al-Shahi Salman R, Beller E, Kagan J, Hemminki E, Phillips RS, Savulescu J, et al. Increasing value and reducing waste in biomedical research regulation and management. Lancet. 2014;383:176-85.

13. Chan AW, Song F, Vickers A, Jefferson T, Dickersin K, Gotzsche PC, et al. Increasing value and reducing waste: addressing inaccessible research. Lancet. 2014;383:257-66.

14. Glasziou P, Altman DG, Bossuyt P, Boutron I, Clarke M, Julious S, et al. Reducing waste from incomplete or unusable reports of biomedical research. Lancet. 2014;383:267-76.

15. loannidis JP, Greenland S, Hlatky MA, Khoury MJ, Macleod MR, Moher D, et al. Increasing value and reducing waste in research design, conduct, and analysis. Lancet. 2014;383:166-75.

16. Glasziou P. The role of open access in reducing waste in medical research. PLoS Med. 2014;11:e1001651.

17. Moher D, Altman DG. Four proposals to help improve the medical research literature. PLoS Med. 2015;12:e1001864.

18. Yordanov Y, Dechartres A, Porcher R, Boutron I, Altman DG, Ravaud P. Avoidable waste of research related to inadequate methods in clinical trials. BMJ. 2015;350:h809.

19. Ioannidis JP. Why Most clinical research is not useful. PLoS Med. 2016;13:e1002049

20. Lund $H$, Brunnhuber $K$, Juhl C, Robinson K, Leenaars M, Dorch BF, et al. Towards evidence based research. BMJ. 2016;355:15440.

21. Bolland MJ, Grey A. A case study of discordant overlapping meta-analyses: vitamin d supplements and fracture. PLoS One. 2014;9:e115934.

22. Elliott JH, Turner T, Clavisi O, Thomas J, Higgins JP, Mavergames C, et al. Living systematic reviews: an emerging opportunity to narrow the evidence-practice gap. PLoS Med. 2014;11:e1001603.

23. Tai V, Grey A, Bolland MJ. Results of observational studies: analysis of findings from the nurses' health study. PLoS One. 2014;9:e110403.

24. Grimes DA, Schulz KF. False alarms and pseudo-epidemics: the limitations of observational epidemiology. Obstet Gynecol. 2012;120:920-7.

25. Bolland MJ, Avenell A, Baron JA, Grey A, Maclennan GS, Gamble GD, et al. Effect of calcium supplements on risk of myocardial infarction and cardiovascular events: meta-analysis. BMJ. 2010;341:c3691.
26. Bolland MJ, Grey A, Avenell A, Gamble GD, Reid IR. Calcium supplements with or without vitamin D and risk of cardiovascular events: reanalysis of the Women's Health Initiative limited access dataset and meta-analysis. BMJ. 2011:342:d2040.

27. Bristow SM, Bolland MJ, Maclennan GS, Avenell A, Grey A, Gamble GD, et al. Calcium supplements and cancer risk: a meta-analysis of randomised controlled trials. Br J Nutr. 2013;110:1384-93.
Ready to submit your research? Choose BMC and benefit from:

- fast, convenient online submission

- thorough peer review by experienced researchers in your field

- rapid publication on acceptance

- support for research data, including large and complex data types

- gold Open Access which fosters wider collaboration and increased citations

- maximum visibility for your research: over $100 \mathrm{M}$ website views per year

At BMC, research is always in progress.

Learn more biomedcentral.com/submissions 